\title{
A Drenagem Linfática no Pós-Operatório Imediato de Cirurgia de Membros Superiores
}

\author{
Lymphatic Drainage in Immediate Post-Operative Upper Limb Surgery \\ Drenaje Linfático en Cirugía Inmediata Post-Operatoria de Miembro Superior
}

Recebido: 29/11/2021 | Revisado: 07/12/2021 | Aceito: 08/12/2021 | Publicado: 16/12/2021

\author{
Ana Paula dos Reis Rocha Coutinho \\ ORCID: https://orcid.org/0000-0002-4280-6067 \\ Faculdade Independente do Nordeste, Brasil \\ E-mail: anapaularrc@hotmail.com \\ Camila Sabino de Souza \\ ORCID: https://orcid.org/0000-0002-4248-2461 \\ Faculdade Independente do Nordeste, Brasil \\ E-mail: Camila20000sabino@gmail.com \\ Matheus Santos Marques \\ ORCID: https://orcid.org/0000-0001-9734-1001 \\ Faculdade Independente do Nordeste, Brasil \\ E-mail: matheusmarques@fainor.com.br
}

\begin{abstract}
Resumo
Introdução: A drenagem linfática pode ser definida como uma técnica de massagem especializada, representada por um conjunto de manobras que visam drenar o excesso de líquido acumulado no interstício. A drenagem linfática manual é uma técnica onde manobras são realizadas para o melhor resultado no pós-operatório imediato de pacientes submetidos a procedimentos cirúrgicos, não sendo usada só para fins estéticos, mas também para prevenção e tratamento de patologias. O presente trabalho tem por escopo demonstrar os benefícios da drenagem linfática no pósoperatório imediato de cirurgia de membros superiores, revisando a anatomia e fisiologia do sistema linfático, verificando o efeito da Drenagem linfática na diminuição ou abolição da dor e analisando os efeitos da drenagem linfática no edema. Objetivo: Tendo como objetivo principal identificar a importância da drenagem linfática manual no tratamento pós-operatório. Método: A pesquisa foi uma revisão bibliográfica de artigos científicos, DeCS, Google acadêmico e Scielo. Conclusão: A Drenagem Linfática Manual no pós-operatório dos membros superiores promove resultados eficazes e satisfatórios tendo uma recuperação excelente trazendo assim uma melhora do edema e do hematoma e consequentemente diminuir a dor relatada pela maioria dos pacientes.
\end{abstract}

Palavras-chave: Edema; Drenagem linfática manual; Estética.

\begin{abstract}
Introduction: Lymphatic drainage is a specialized massage technique which includes a set of maneuvers that aim to drain excess fluid accumulated in the interstitium. Manual lymphatic drainage is a technique which is performed for the best results during the immediate post-operative period of patients undergoing surgical procedures. It is not only used for aesthetic purposes but also for the prevention and treatment of pathologies. The present work aims to demonstrate the benefits of lymphatic drainage in the immediate post-operative period of upper limb surgery, reviewing the anatomy and physiology of the lymphatic system, verifying the effect of lymphatic drainage in reducing or eliminating pain and analyzing the effects of lymphatic drainage on edema. Objective: The objective main is to identify the importance of manual lymphatic drainage in post-operative treatment. Method: The research method was a literature review of scientific articles, DeCS and academic Google. Conclusion: o comply with the Manual Lymphatic Drainage in the upper limbs postoperatively, they provide effective and satisfactory results, with an excellent recovery, thus bringing an improvement in edema and hematoma and consequently reducing pain reported by most patients.
\end{abstract}

Keywords: Edema; Manual lymphatic drainage; Aesthetics.

\section{Resumen}

Introducción: El drenaje linfático se puede definir como una técnica de masaje especializada, representada por un conjunto de maniobras que tienen como objetivo drenar el exceso de líquido acumulado en el intersticio. El drenaje linfático manual es una técnica donde se realizan maniobras para el mejor resultado en el postoperatorio inmediato de los pacientes sometidos a procedimientos quirúrgicos, utilizándose no solo con fines estéticos, sino también para la prevención y tratamiento de patologías. El presente trabajo tiene como objetivo demostrar los beneficios del drenaje linfático en el postoperatorio inmediato de cirugía de miembro superior, revisando la anatomía y fisiología del sistema linfático, verificando el efecto del drenaje linfático en la reducción o abolición del dolor y analizando los efectos del drenaje linfático en edema. Objetivo: El objetivo principal es identificar la importancia del drenaje linfático manual en el tratamiento postoperatorio. Método: El método de investigación fue una revisión de la literatura de artículos 
científicos, DeCS, Academic Google y Scielo. Conclusión: Para concluir, el Drenaje Linfático Manual en miembros superiores en el postoperatorio promueve resultados efectivos y satisfactorios, con una excelente recuperación, trayendo así una mejoría en el edema y hematoma y consecuentemente disminuyendo el dolor reportado por la mayoría de los pacientes.

Palabras clave: Edema; Drenaje linfático manual; Estética.

\section{Introdução}

A drenagem linfática manual foi iniciada nos anos 1930 pelo dinamarquês Emil Vodder e de sua esposa, Estrid Vodder.

Segundo Piccinini, (2011), estas técnicas, tanto de Vodder quanto de Leduc, têm como diferença o movimento. Vodder pratica movimentos circulares de bombeio e rotatórios, enquanto Leduc pratica movimentos mais restritos. As técnicas relacionam três categorias de manobras: Capitação, reabsorção e evacuação.

A DLM é uma técnica de movimentos finos, suaves, superficiais, tendo como direção centrípeta de proximal para distal. Tem como função a evacuação de toxinas e restos metabólicos, drenando os líquidos excedentes das células. "Os movimentos devem ser lentos, delicados e rítmicos, reproduzindo o bombeamento fisiológico, evitando, assim, o rompimento dos vasos" como relata Mauad (2008).

Para um tratamento de pós-operatório adequado para cada caso, deve ser realizado a anamnese visando reconhecer as particularidades de cada paciente, cirurgião e da técnica cirúrgica executada. Nesse contexto, devem ser avaliados quais os tecidos envolvidos e profundidade atingida, o motivo pelo qual o paciente buscou a cirurgia, estágio da cicatrização, o tipo de tratamento que será aplicado, bem como sua duração e frequência de realização, avaliando possíveis riscos e individualidades do paciente, para obtenção de bons resultados (Sdregotti et al 2016).

A aplicação da drenagem linfática no pós-operatório faz com que se canalize o edema a um gânglio próximo a lesão, como uma forma para não haver encharcamento da cicatriz e consequentemente o aumento de edema, haja vista que dependendo da cirurgia e a sua localização onde terá uma secção, vasos são lesionados, prejudicando a eliminação dos líquidos em demasia. (Macedo \& Oliveira, 2010).

"A drenagem não oferece risco algum para o paciente em pós-operatórios de cirurgias plásticas, somente se for mal aplicada empregando muita força, rapidez excessiva ou direção errada. Não há limites para utilização e as técnicas de aplicação para as sequelas pós-cirúrgicas podem ser baseadas na drenagem reversa que consiste em direcionar o edema a um gânglio proximal a lesão como uma via alternativa para não haver encharcamento da cicatriz e aumento de edema, já que dependendo da cirurgia onde há uma secção, vasos são lesionados, dificultando assim a eliminação dos líquidos excedentes" (Macedo \& Oliveira, 2010:191).

Ensina Brito (2021) que o edema é uma disfunção consistente no excesso anormal de líquido nas cavidades corporais devido ao mau funcionamento do sistema linfático. Trata-se de uma solução aquosa de sais e proteínas do plasma, e sua composição vai depender da causa que levou a desenvolver essa patologia. Quando o líquido se acumula no corpo, ele se torna um edema generalizado, citando Ozolins (2018).

Para Foldi (2012), a DLM deve ser iniciada 72 horas após a cirurgia, para a prevenção de possíveis fibroses. Esses movimentos têm que seguir a circulação linfáticas sendo rítmicas, suaves e lentas.

\footnotetext{
${ }^{1}$ Acadêmico do Curso de Estética e Cosmetologia, da Faculdade Independente do Nordeste - FAINOR.

${ }^{2}$ Acadêmico do Curso de Estética e Cosmetologia, da Faculdade Independente do Nordeste - FAINOR.

${ }^{3}$ Orientador, Professor do curso DE Estética e Cosmetologia da Faculdade Independente do Nordeste - FAINOR.
} 
À vista disso, a drenagem linfática é uma técnica grandemente utilizada e indicada pelos profissionais, por dedicar ao paciente qualidade de vida e prevenção de prováveis sequelas pós-operatória, além de oferecer melhora do edema, cicatrizes, entre outros.

Por isso a importância da drenagem linfática ser realizada por profissional capacitado da área de estética, pois vai ajudar na redução desses eventos clínicos, abreviando o processo de recuperação do pós-operatório, prevenindo, assim, futuras complicações, conforme afirma o autor Utiyama (2003.)

Frente ao exposto, esse estudo tem como objetivo analisar os benefícios da drenagem linfática no pós-operatório imediato de membros superiores.

\subsection{Indicações e Contra Indicações da Drenagem Linfática Manual}

A drenagem linfática manual é indicada em quase todo tipo de pós-operatório de cirurgia plástica, dentre elas esta a cirurgias de face, mamas, abdominoplastias, lipoaspiração, entre outras, como relata o Migotto e Simões (2013) e Brandão et al (2010).

A drenagem linfática manual é indicada em quase todo tipo de pós-operatório de cirurgia plástica, dentre elas esta a cirurgias de face, mamas, abdominoplastias, lipoaspiração, entre outras, como relata o Migotto e Simões (2013) e Brandão et al (2010).

Já segundo Guirro (2002), citado por Soares (2012), a DLM deve ser indicada de acordo com as disfunções citadas pelo paciente, que podem ser cicatrizes, hematomas, dor, edemas, diminuição de sensação, tensão muscular, vez que existem algumas comorbidades em que a drenagem linfática não é indicada, como: distúrbios circulatórios, hiperestesia, processo infeccioso, tumores malignos e fragilidade capilar.

"Um profissional deve ser capaz de identificar as indicações e contraindicações, que uma indicação é benéfica ao paciente e que acentue a saúde. Há contraindicação quando a pratica se torna perniciosa, trazendo malefícios aos pacientes submetidos à técnica proposta". (Fritz 2002, citado por Soares, 2012:78).

\section{Metodologia}

Trata-se de uma revisão integrativa de literatura. Deste modo, a questão norteadora do estudo parte do seguinte questionamento: "Quais são os benefícios e vantagens da drenagem linfática no pós-operatório imediato de cirurgia de membros superiores?".

Foram utilizados como base de dados: Google acadêmico e a Biblioteca Virtual Scielo, e para realização de buscas os seguintes descritores, (Dec's), Edema, Cirurgia, Estética, utilizando os operadores booleanos “AND" e "OR" entre os termos.

Como critérios de inclusão foram adotados artigos publicados entre 2003 a 2021 no idioma português com pertinência temática e foram excluídos do estudo teses, dissertações, livros, capítulos de livros e artigos que não apresentavam disponibilidade do seu conteúdo integral online.

Dentro dos critérios de inclusão foram analisados inicialmente 53 artigos, sendo que após a aplicação do critério de exclusão foram selecionados 32 .

A análise de dados obtida foi realizada através da construção de uma tabela com os seguintes dados: ano de publicação, Autor, Tema, Objetivo, Métodos, resultados. 


\section{Resultados}

Quadro 1. Artigos selecionados.

\begin{tabular}{|c|c|c|c|c|c|}
\hline Ano & Autores & Tema & Objetivo & Métodos & Resultados \\
\hline 2007 & $\begin{array}{l}\text { DA SILVA, } \\
\text { Giselle } \\
\text { Oliveira1. DE } \\
\text { SOUZA, } \\
\text { Flaviano } \\
\text { Gonçalves } \\
\text { Lopes }^{2} \text {. }\end{array}$ & $\begin{array}{l}\text { Os benefícios } \\
\text { da drenagem } \\
\text { linfática manual } \\
\text { no pós- } \\
\text { operatório } \\
\text { imediato de } \\
\text { lipoaspiração }\end{array}$ & $\begin{array}{l}\text { Demonstrar que a } \\
\text { DLM, resulta na } \\
\text { melhora da dor, e } \\
\text { diminuindo } \\
\text { hematomas }\end{array}$ & $\begin{array}{l}\text { Foram realizadas pesquisas com revisão } \\
\text { bibliográfica em fontes primárias e } \\
\text { secundárias do tipo qualitativo. } \\
\text { Apresentado de maneira descritiva, com o } \\
\text { objetivo, de relatar alguns dos benefícios } \\
\text { da drenagem linfática Manual no pós- } \\
\text { operatório de lipoaspiração. A revisão de } \\
\text { livros acadêmicos e artigos científicos tem } \\
\text { como referência os trabalhos publicados a } \\
\text { partir de 2007. Também foi utilizado o } \\
\text { Portal BioCursos, a base de dados da } \\
\text { biblioteca digital SCIELO (Scientific } \\
\text { Eletronic Library Online) e Google } \\
\text { acadêmico }\end{array}$ & $\begin{array}{l}\text { Após analise dos artigos e bibliografias que } \\
\text { relatam os benefícios da drenagem linfática } \\
\text { manual como um tratamento pós-operatório } \\
\text { de cirurgias plásticas e lipoaspiração, } \\
\text { percebe-se que sua principal característica } \\
\text { está em melhorar o estado do paciente, e que } \\
\text { ainda é uma das principais técnicas } \\
\text { recomendadas e utilizadas no pós-operatório. }\end{array}$ \\
\hline 2011 & $\begin{array}{l}\text { RAMOS, } \\
\text { João Erfon } \\
\text { Almeida. }\end{array}$ & $\begin{array}{l}\text { Abdominoplasti } \\
\text { a com } \\
\text { lipoaspiração e } \\
\text { descolamento. } \\
\text { Mínimo }\end{array}$ & $\begin{array}{l}\text { Mostrar a eficácia e } \\
\text { benefícios da DLM } \\
\text { após a cirurgia da } \\
\text { Abdominoplastia }\end{array}$ & $\begin{array}{l}\text { Foram realizados } 483 \text { abdominoplastias no } \\
\text { período de junho de } 2001 \text { a julho de } 2010 \text {, } \\
\text { sendo } 480 \text { pacientes do sexo feminino e } 3 \text {, } \\
\text { do masculino, com idades variando de } 24 \\
\text { a } 61 \text { anos. Pela base da pesquisa nesse } \\
\text { artigo foram realizadas, } \\
\text { miniabdominoplastias, } 35 \\
\text { abdominoplastias }\end{array}$ & $\begin{array}{l}\text { Concluiu-se que a utilização da lipoaspiração } \\
\text { associada à abdominoplastia1,7,9, com } \\
\text { descolamento mínimo e acrescida da } \\
\text { ressecção medial infraumbilical e da } \\
\text { pubectomia parcial, permite a obtenção de } \\
\text { resultados estéticos mais favoráveis, levando } \\
\text { à melhor delineação do contorno corporal da } \\
\text { paciente, bem como reduzindo as } \\
\text { complicações da abdominoplastia clássica, } \\
\text { tais como seromas, necroses e alta incidência } \\
\text { de lipoaspirações secundárias. }\end{array}$ \\
\hline 2017 & $\begin{array}{l}\text { SAWNTSY, } \\
\text { Jane Regina } \\
\text { Frewyd }{ }^{1} \text {. DE } \\
\text { SOUZA, } \\
\text { Flaviano } \\
\text { Golsalves } \\
\text { Lopes }^{2} \text {. }\end{array}$ & $\begin{array}{l}\text { A eficácia da } \\
\text { drenagem } \\
\text { linfática manual } \\
\text { na pós de } \\
\text { Rinoplastia. }\end{array}$ & $\begin{array}{l}\text { Mostrar o benefício } \\
\text { imediato, podendo } \\
\text { ser realizada no } \\
\text { segundo dia após a } \\
\text { cirurgia, e o paciente } \\
\text { que foi operado tem } \\
\text { um alivio } \\
\text { significativo na } \\
\text { região após ser } \\
\text { realizada a DLM. }\end{array}$ & $\begin{array}{l}\text { Foram realizados levantamentos } \\
\text { bibliográficos no período de fevereiro de } \\
2017 \text { a setembro de } 2017 \text {, junto às bases } \\
\text { de dados com dez artigos científicos e } \\
\text { revistas científicas a partir das seguintes } \\
\text { palavras chaves: drenagem linfática, } \\
\text { edema, pós-operátorio, rinoplastia. Dentre } \\
\text { as linguagens utilizadas selecionamos } \\
\text { artigos em português e inglês. }\end{array}$ & $\begin{array}{l}\text { Esse estudo conclui que há benefícios } \\
\text { positivos para o paciente, e, se o este tiver } \\
\text { um acompanhamento fisioterapêutico que } \\
\text { realize a drenagem linfática manual no pós- } \\
\text { operatório imediato, sua recuperação pode } \\
\text { ser mais rápida, pois melhora o quadro } \\
\text { doloroso, ajuda na circulação local e } \\
\text { hidratação cutânea, pois o paciente de } \\
\text { rinoplastia sai da sala de cirurgia com o rosto } \\
\text { edemaciado, dolorido e com a sensação de } \\
\text { ter sido muito machucado. }\end{array}$ \\
\hline 2018 & $\begin{array}{l}\text { NUNES, } \\
\text { Jéssica } \\
\text { Espínola }\end{array}$ & $\begin{array}{l}\text { A eficácia da } \\
\text { drenagem } \\
\text { linfática manual } \\
\text { no linfedema } \\
\text { pós- } \\
\text { mastectomia }\end{array}$ & $\begin{array}{l}\text { Analisar e expor os } \\
\text { benefícios da DLM } \\
\text { e descrever as suas } \\
\text { fases no pós- } \\
\text { mastectomia. }\end{array}$ & $\begin{array}{l}\text { Foram utilizados bases de pesquisa } \\
\text { SciELO e Bireme, as palavras chaves e/ou } \\
\text { termos utilizados para pesquisa foram } \\
\text { fisioterapia dermatofuncional, drenagem } \\
\text { linfática manual, sistema linfático, câncer } \\
\text { de mama, linfedemas e pós-operatório. Os } \\
\text { idiomas dos artigos foram em Inglês e em } \\
\text { Português, os anos de publicação entre } \\
1973 \text { e } 2017 \text {. }\end{array}$ & $\begin{array}{l}\text { Concluiu-se que o tratamento } \\
\text { fisioterapêutico no câncer de mama se torna } \\
\text { importante para evitar problemas como o } \\
\text { linfedema, principal complicação estudada } \\
\text { neste artigo, observando-se diminuição do } \\
\text { mesmo através de técnicas bem aplicadas } \\
\text { como a drenagem linfática manual (DLM) } \\
\text { podendo ou não ser associada a outras } \\
\text { técnicas para melhores resultados nas } \\
\text { pacientes pós mastectomizadas com } \\
\text { presença de linfedemas. }\end{array}$ \\
\hline 2020 & $\begin{array}{l}\text { PRADO, } \\
\text { alice } \\
\text { Souza }{ }^{1}, \text { MACI } \\
\text { EL, Barbara } \\
\text { Figueiredo }{ }^{2}, \\
\text { TEIXEIRA, } \\
\text { Fagundes } \\
\text { Soares }{ }^{3} \text {, } \\
\text { FACUNDES, } \\
\text { Guilia Rivele } \\
\text { Souza }\end{array}$ & $\begin{array}{l}\text { Os benefícios } \\
\text { da drenagem } \\
\text { linfática pós- } \\
\text { mastectomia }\end{array}$ & $\begin{array}{l}\text { Mostrar a eficácia e } \\
\text { benefícios da DLM } \\
\text { no pós-mastectomia. }\end{array}$ & $\begin{array}{l}\text { Foram realizado uma pesquisa } \\
\text { bibliográfica sobre o tema em livros e nas } \\
\text { bases de dados do google acadêmico e } \\
\text { Scielo (Scientifc Eletronic Library } \\
\text { Online), no idioma português.pesquisa foi } \\
\text { realizada por meio de levantamento } \\
\text { bibliográfico em livros técnicos, consulta á } \\
\text { internet e artigos publicados por } \\
\text { pesquisadores da área de saúde. Os artigos } \\
\text { foram selecionados a fim de obter } \\
\text { informações embasadas e consistentes no } \\
\text { que diz respeito ao benefício da drenagem } \\
\text { linfática manual para o paciente em } \\
\text { particular as mulheres que são acometidas } \\
\text { pelo câncer de mama, sendo necessário } \\
\text { realizar a mastectomia. }\end{array}$ & $\begin{array}{l}\text { Concluiu-se que a drenagem linfática manual } \\
\text { se torna uma manobra muito importante no } \\
\text { tratamento de pacientes que fizeram a } \\
\text { mastectomia, pois, evita problemas como o } \\
\text { linfedema, fibroses e hematomas, principal } \\
\text { complicação estudada neste artigo, onde } \\
\text { haverá diminuição do fluído através da } \\
\text { técnica bem aplicada da drenagem e } \\
\text { podendo também ser associada a outras } \\
\text { técnicas para melhores resultados nas } \\
\text { pacientes pós mastectomizadas. }\end{array}$ \\
\hline
\end{tabular}

Fonte: Autores. 


\section{Discussão}

Atualmente a drenagem linfática dos membros superiores no pós-operatório está sendo indicada por vários profissionais da saúde devido aos seus benefícios concernentes na melhora significativa dos pacientes, principalmente do que diz respeito às complicações que por ventura poderiam ocorrer nos primeiros momentos após o procedimento cirúrgico.

Segundo Alencar (2011), a utilização da DLM traz mudanças significativas nos procedimentos cirúrgicos, em especial no processo de criação de barreiras protetoras das lesões, o que ajuda no processo de recuperação dos tecidos vez que facilita o processo de cicatrização.

Para Borges (2006), a DLM, após a cirurgia, faz com que haja melhoras no incomodo da dor, tendo em vista a redução do acúmulo tecidual, fazendo que a sensibilidade cutânea retorne de forma mais rápida.

Parafraseando Borges (2006), ao se realizar intervenções cirúrgicas, ocorre a destruição de vasos e nervos, o que causa edemas, dores e diminuição da sensibilidade cutânea, gerando dor ao paciente. Isso pode ser evitado realizando drenagem linfática manual após essas intervenções estéticas ou reparadoras.

Esse tipo de terapia no pós-operatório faz que haja melhoras simples como no desconforto até mais complexos, melhorando quadro álgico.

Já Sanches (2002) afirma que o único procedimento que é realizado normalmente após as 48 horas da cirurgia é a DLM, deixando o paciente restrito a movimentos até 21 dias após a cirurgia, com o risco de descolamento do tecido epitelial.

De acordo com Ribeiro (2003), a drenagem linfática manual é necessária e deve ser feita o mais rápido possível, pois faz com que tenha maior absorção de líquidos dos capilares sanguíneos e linfáticos onde ocorreu a lesão.

Ribeiro (2000) também relata que, visando uma possível redução de edemas e hematomas, a pressão exercida na massagem deve atuar diretamente na circulação, e se deslocar de forma centrípeta, fazendo com que haja um aumento da reabsorção do líquido para os capilares venosos.

De acordo com Winter (2005), o profissional da estética é indispensável para que haja contribuição no tratamento pósoperatório, realizando o processo de DLM como o principal procedimento, visando a diminuição do edema. Com isso, a DLM é realizada com o intuito de estimular a pele a recuperar seu aspecto natural. Ela capta o líquido intersticial e devolve à circulação sanguínea, que através do movimento suave, lento e rítmico, promove melhora da oxigenação e nutrição celular, consequentemente promovendo a desintoxicação das células.

Portanto, de acordo com os autores citados acima, o tratamento da Drenagem Linfática no Pós-operatório de membros superiores se torna importante para evitar problemas futuros após as cirurgias realizadas, trazendo ao paciente o alívio de dores, absorção de hematomas e seromas, resultados estes que serão alcançados através de técnicas bem aplicadas.

\section{Conclusão}

A Drenagem Linfática Manual é o recurso que esta sendo mais indicado e valorizado em todos os pós-operatórios, conforme os cirurgiões plásticos e autores relatados a cima. Esta técnica é amplamente utilizada para tratar diversas patologias, que irá atuar na melhora da cicatrização da lesão, além de tratar edemas e o alívio de dores causados pela cirurgia, bem como ajudará na absorção de hematomas e seromas, tudo isso traduzindo em benefícios imediatos ao paciente.

Sendo assim, o profissional de Estética que irá atender seus pacientes do pós-cirúrgicos é fundamental para completa reabilitação destes. Com ajuda do profissional de Estética, é possível a redução de edemas e hematomas, com a consequente diminuição das dores relatadas pela maioria dos pacientes, o que promove resultados eficazes e satisfatórios.

Uma vez explanados os benefícios que a DLM traz, é de muito proveito que os futuros trabalhos relacionados a esse tema relatem e aprofundem a importância desta terapia manual. Salutar, também, que a DLM seja abordada como protocolo de 
tratamento de outras patologias, tais como: circulação sanguínea com retorno comprometido, tratamento pós cirurgia plástica, cicatrizes, dentre outras.

Deste modo, é importante que haja mais estudos clínicos, a fim de se usar cada vez mais os protocolos de drenagem nas mais diversas enfermidades e aperfeiçoar o trabalho daqueles que usam esse tratamento.

\section{Referências}

Alencar, T. P. \& Meja, D. P. M. (2011). A influência da drenagem linfática manual em pós-operatório imediato de cirurgia vascular de membros inferiores. Estudo de Caso. https://portalbiocursos.com.br/ohs/data/docs/14/24_-_A_influYncia_da_drenagem_linfYtica_manual_no_pYs_operatYrio_imediato_de_cirurgia_vascular_de_membros_inferiores.pdf

Alves, B. R., Schock, J. W., Scherf, G., \& Gerhardt, L. G. (2012). Utilização Da Técnica De Drenagem Linfática Manual No Pós-Operatório De Lipoaspiração. Salão do Conhecimento, https://publicacoeseventos.unijui.edu.br/index.php/salaoconhecimento/article/view/17274/15956.

Amaral, A. C. S. et. al. (2014) Os Corpos da Cirurgia Plástica: Os Discursos de Mulheres Sobre Corpo, Aparência e Atividade Física. http://www.fef.unicamp.br/hotsites/imagemcorporal2010/cd/anais/trabalhos/portugues/Area2/|C2-02.pdf.

Borges, F. dos S. (2006). Dermato-Funcional: Modalidades Terapêuticas nas Disfunções Estéticas. Phorte.

Brandão, D. S. M., et al. (2010). Avaliação da Técnica de Drenagem Linfática Manual no Tratamento do Fibro Edema Geloide em Mulheres. ConScientae Saúde, 1(4), http://www.redalyc.org/pdf/929/92921672010.pdf.

Campos, F. M. (2016). Drenagem Linfática no Pós-Operatório de Mamoplastia: Uma Revisão Bibliográfica. https://portalbiocursos.com.br/ohs/data/docs/199/9-Drenagem_linfYtica_no_pYs-operatYrio_de_mamoplastia_uma_revisYo_bibliogrYfica.pdf.

de Brito, P. K. S., Angelim, C. C., \& Casseb, S. M. M. (2021). Uma Revisão Sistemática sobre os Benefícios da Drenagem Linfática Manual no Tratamento do Edema em Membros Inferiores. Research, Society and Development, 10(4), e14810413968-e14810413968

da Silva, A. M. D. M. A. (2018). A Importância da Drenagem Linfática no Tratamento Estético e Terapêutico no Pós-Operatório de Lipoaspiração. https://portalbiocursos.com.br/ohs/data/docs/234/22-A_importYncia_da_drenagem_linfYtica_no_tratamento_estYtico_e_terapYutico_no_pYsoperatYrio_de_LipoaspiraYYo..pdf

da Silva, G. O., \& de Souza, F. G. L. (2016). Os Benefícios da Drenagem Linfática Manual no Pós-Operatório Imediato de Lipoaspiração. https://portalbiocursos.com.br/ohs/data/docs/234/26-Os_benefYcios_da_drenagem_linfYtica_manual_no_pYs-operatYrio_imediato_de_lipoaspiraYYo.pdf.

da Silva, L. A., \& Mejia, D. P. M. (2011). A Importância da Drenagem Linfática Manual no Pós-Operatório de Lipoaspiração e Abdominoplastia. Revisão de Literatura https://portalbiocursos.com.br/ohs/data/docs/39/05_-_A_ImportYncia_da_Drenagem_LinfYtica_Manual_no_PYsOperatYrio_de_LipoaspiraYYo_e_Abdominoplastia.pdf.

Foldi, M. \& Strobenreuther, R. (2012). Princípios da Drenagem Linfática. Manole. 4 Ed.

Gil, A. C. (2002). Como Elaborar Projetos de Pesquisa. 4(175).: Atlas.

GUYTON, A. C. (1997). Tratado de Fisiologia Médica. (9a ed.), Guanabara Koogan.

Lays B. P. \& Dayana P. M. M. Benefícios da Drenagem Linfática Manual no Tratamento Pós-Operatório Imediato de Abdominoplastia. https://portalbiocursos.com.br/ohs/data/docs/98/141-BenefYcios_da_drenagem_linfYtica_manual_no_tratamento_pYsoperatYrio_imediato_de_abdominoplastia.pdf.

Leduc, A. \& Leduc, O. (2007). Drenagem Linfática Teoria e Prática. (3a ed.), Manole.

Macedo, A. C. B. \& Oliveira, S. M. (2010). A Atuação da Fisioterapia no Pré e Pós-Operatorio de Cirurgia Platica Corporal: Uma Revisão de Literatura. Cadernos da Escola de Saúde, 1(4). https://portalbiocursos.com.br/ohs/data/docs/98/141-BenefYcios_da_drenagem_linfYtica_manual_no_tratamento_pYsoperatYrio_imediato_de_abdominoplastia.pdf.

Machado, D. (2009). Cirugia plástica. 2009. Website Davi Machado. http://www.davimachado.com.br/Downloads/jornalDaviMachado.pdf.

Marx, A. G. \& Camargo, M.C. (1986). Fisioterapia no Edema Linfático. Paramed.

Mauad, R. (2008). Estética e Cirurgia Plástica: Tratamento no Pré e Pós-Operatório. (3a ed.), SENAC.

Moraes, L. M. (2008). Resgate da Auto-Estima. Revista Ética e Estética. 9(35). https://www.rdobrasil.com.br/revista/pdf/revista_ed35.pdf.

Moura, V. M. \& Mejia, D. P. M. (2014). A Importância da Técnica de Drenagem Linfática Manual no Tratamento Pós-Operatório de Abdominoplastia. https://portalbiocursos.com.br/ohs/data/docs/18/112_-_A_importYncia_da_tYcnica_de_drenagem_linfYtica_manual_no_tratamento_pYsoperatYrio_de_abdominoplastia.pdf.

Migotto, J. S. \& Simões, N. D. P. (2013). Atuação Fisioterapêutica Dermato Funcional no Pós-Operatório de Cirurgias Plásticas. Revista Eletrônica Gestão e Saúde, 4(1). http://164.41.147.155/gestaoesaude/index.php/gestaoesaude/article/view/263.

Nunes, J. E. (2018). A Eficácia da Drenagem Linfática Manual no Linfedema Pós Mastectomia. https://bibliodigital.unijui.edu.br:8443/xmlui/bitstream/handle/123456789/5304/J\%C3\%A9ssica\%20Esp\%C3\%ADndola\%20Nunes.pdf?sequence=1. 
Ozolins, B. C. et al (2018). Drenagem Linfática Clássica: Revisão de Literatura. Revista Saúde em Foco, (10), 319-323,

Pessoa, L. B., \& Mejia, D. P. M. Benefícios da Drenagem Linfática Manual no Tratamento Pós-Operatório Imediato de Abdominoplastia. Revista de Escolha.

Piccnini, A. M. et.al. (2009) Redução do Edema em Membros Inferiores Através da Drenagem Linfática Manual: Um Estudo de Caso. Revista Inspirar. 1(2).

Prado, A. S., Maciel, B. F., Teixeira, F. S. \& Facundes, G. R. S. (2020). Os Benefícios da Drenagem Linfática Pós Mastectomia. Revista de psicologia. 14(52), 362-373. 10.14295/idonline.v14i52.2720. https://idonline.emnuvens.com.br/id/article/viewFile/2720/4357.

Ramos, J. E. A. (2011). Abdominoplastia com Lipoaspiração e Descolamento Mínimo. Revista Brasileira de Cirurgia Plástica. 26(1), 116-120. https://doi.org/10.1590/S1983-51752011000100021. https://www.scielo.br/j/rbcp/a/8S9cFfMrWkgF6kb3tpGMw4r/?format=pdf\&lang=pt.

Sanches, O. (2002). Pré e Pós Cirúrgico Plástica. Personalité - Revista Profissional Multidisciplinar, (24), 11-17.

Santos, G. N. \& Alves, I. C. P. (2017). Complicações da cicatriz pós-mamoplastia com inclusão de implante de silicone associada à mastopexia: Estudo de caso. Revista Científica Posteriori. Cirurgia Plástica. 3.

Sawntsy, J. R. F., \& de Souza, F. G. L. Os Benefícios da Drenagem Linfática Manual no Pós-Operatório de Rinoplastia. https://portalbiocursos.com.br/ohs/data/docs/234/28-Os_benefYcios_da_Drenagem_LinfYtica_Manual_no_PYs_-_OperatYrio_da_Rinoplastia.pdf.

Soares, R. G. (2012). Drenagem Linfática Manual como Coadjuvante no Pós-Operatório de Abdominoplastia. Revista Presciência, (5). http://www.faculdadesaomiguel.com.br/Presciencia5A.pdf.

Utiyama, Y. et.al. (2003). Estudo retrospectivo de 288 lipoaspirações realizadas no serviço de dermatologia do Hospital do Serviço Público Municipal de São Paulo. Anais brasileiros de dermatologia, 78(4), 435-442. 